\title{
Biological Factors in Metastatic Dissemination of Head and Neck Squamous Cell Carcinomas
}

\author{
Gabriela Alejandra Iasi ${ }^{1}$ and Guido Valente ${ }^{2 *}$ \\ ${ }^{1}$ Laboratory of Pathology, Ospedale Sant'Andrea, Vercelli, Italy \\ ${ }^{2}$ Dipartimento Di Medicina Traslazionale, UPO School Of Medicine, Vercelli, Italy \\ *Corresponding Author: Guido Valente, Dipartimento Di Medicina Traslazionale, UPO School Of Medicine, Vercelli, Italy.
}

Received: September 16, 2019; Published: October 17, 2019

DOI: $10.31080 / A S M S .2019 .03 .0441$

\begin{abstract}
Head and neck squamous cell carcinoma (SCC) is the sixth most common cancer in the world, accounting for approximately $6 \%$ of all cancer cases, being a significant cause of morbidity and mortality worldwide (about $2 \%$ of cancer related deaths). It is ubiquitarian in upper respiratory and digestive tract, both areas characterized by a marked anatomical complexity and profound differences, for instance in terms of structure, lymphatic drainage and vascularization: this implies that each global consideration about prognosis of these tumors may not be conclusive or satisfactory, since the diffusion is at least partially dependent from the site of origin.
\end{abstract}

Keywords: Biological Factors; Metastatic Dissemination; Head and Neck

\section{Introduction}

Moreover, in at least two sites (nose/paranasal sinuses and salivary glands), anatomical complexity is strengthened by the variability of histological subtypes of malignant tumors, other than SCC, potentially occurring here. It is well established that aggressiveness of head and neck tumors is manifested mainly as local recurrences and lymph node metastases more than distant diffusion. Many cases of local recurrences are due to a sort of biological "condemnation" of the mucosa known as field cancerization [1], while the pathways of metastatic diffusion are explained by one hand with molecular assets of the tumor and by the other hand by the complex interactions between neoplastic tissue and tumor microenvironment [2].

In this review we will refer at first to the well-known molecular pathways of local diffusion and metastasis of HNSCC, concerning both field cancerization and tissue microenvironment; by ending, we will report some recent results of translational research about this topic.

\section{Molecular mediators of lymph node metastasis in HNSCC}

Breakdown of cell-to-cell adhesion: E-cadherine /catenine

Cadherine family of cell adhesion molecules mediates homotypic cell-to-cell adhesion within epithelial cells in a calcium dependent way, by means of its extracellular domain and forming complexes with actin cytoskeleton through cytoplasmic catenine.

Downregulation or dysfunction of cadherine or catenine produces a loss of integrity in the cell-to- cell adhesion mechanism and subsequent dissociation of the epithelial cells, being a prerequisite for active migration and metastasis.

In HNSCC with lymph node metastases an association between loss of E-cadherin and catenin complex and presence of metastasis has been demonstrated. Expression of E-cadherin was significantly lost in the metastatic tissue in comparison to primary tumor and surrounding normal mucosa [3]. Reduced expression or aberrant localization of $\alpha$-, $\beta$ and $\delta$ - catenin may be a useful tool to predict a risk of lymph node metastasis [4]. 
Integrins and CD44

Integrins are a family of heterodimeric transmembrane glycoproteins with function of cell surface receptors, composed of noncovalently associated $\alpha$ and $\beta$ subunits involved in intercellular and cellular-extracellular matrix attachment, contributing to a bidirectional exchange between extra and intracellular environment.

Aberrant integrin expression has been implicated in HNSCC tumour invasion and metastasis. The most commonly expressed integrin in the basal layer of the normal squamous cell epithelia is $\alpha v \beta 6$ and its altered expression has been reported in the development of HNSCC [5].

CD44 is a glycosilated multifunctional cell surface protein that works as receptor for hyaluronic acid in extracellular matrix. Multiple isoforms exist due to a mechanism of post-transcriptional splicing. A predominant loss of expression in some isoforms (v4, v5, v6, v9) has been correlated with lymph node metastases $[6,7]$.

Modulation of extracellular matrix and proteolytic enzymes: matrix metalloproteinases (MMP) and Cathepsin D

Proteolytic enzymes are implicated in the degradation of extracellular matrix components such as fibronectin, collagen, laminin, glycoproteins and proteoglycans, and contribute to favor stromal invasion of tumor cells, as well as vessel intravasation and extravasation.

Matrix metalloproteinases, a family of zinc dependent endopeptidases secreted in an inactivated form, seem mainly involved in the metastatic process. An increased expression and activity of matrix metalloproteinases (main variants involved are MMP-1, MMP-2, MMP-9), associated to overexpression of EGFR, has been evidenced both in HNSCC tumor samples and in their metastases, being predictive of a poor prognosis [8,9].

Cathepsinas are lysosomal endopeptidases overexpressed in various types of human carcinomas. Limited to head and neck area, high levels of Cathepsina-D correlates directly with the presence of lymph node metastases in oral carcinomas [10,11].

Molecular alterations favoring cell motility and tumor cell migration: MET oncogene and EGFR

MET is a proto-oncogene encoding for a tyrosine kinase receptor for Scattered Factor also known as Hepatic Growth Factor (HGF): it promotes cell proliferation, detachment and motility.
Ontogenically activated MET can favour the growth and give invasive and metastatic properties to clones of neoplastic cells, as demonstrated by the high level of mutant alleles of MET found in metastatic lymph nodes of HNSCC, in comparison to the barely detectable level evidenced in the concomitant primary tumors [12].

EGFR (Epidermal Growth Factor Receptor) is a family of transmembrane proteins which includes four members (c-erbB-1/ EGFR, c-erbB-2/Her-2neu, c-erbB-3/Her-3 and c-erbB4/Her-4) and are involved in tumour growth and malignant transformation through a tyrosine kinase activity. The role of the EGFR family in HNSCC has been thoroughly investigated.

Several studies have reported an independent correlation of EGFR members with the presence of nodal metastases and poor clinical outcome, due to cooperative signaling of all EGFR receptor members. They enhance proliferation and invasion of HNSCC cells, by activating the MAPK and PI3K downstream signaling pathways involved in the transcriptional regulation of proteases and cytokines [13].

Biological aspects of the tissue microenvironment (TME) in HNSCC and their influence on aggressiveness and metastatic behavior

Genetic and epigenetic changes of normal squamous epithelium

A number of studies have demonstrated that genetic changes of the normal epithelium in mucosa of upper respiratory and digestive tract are associated to HPV infection (mainly the strains 16, 18 and 31) and follow the same pathway described in the cervical cancer, via E6 and E7 proteins.

In non HPV-related HNSCC, the most frequent epithelial genetic change is mutation of tumor-suppressor gene TP53, present in more than $50 \%$ of cases in malignant and pre-malignant lesions. The mutations of TP53 are associated to:

- Increased migration of cancer associated fibroblasts (CAF), favouring invasiveness and progression of neoplasia [14].

- Increased production of reactive oxygen species (ROS) and reactive nitrogen species (NOS) driving carcinogenesis via NF-kB and promoting abnormal tumor metabolism through the Warburg effect. 
Mutations of NOTCH 1 (transmembrane receptor that regulates cell differentiation and embryonic development) contribute to proliferation and invasiveness through increasing concentration of TNF acting on Slug and Twist and its regulation over epithelial to mesenchymal transition (EMT) [15].

EGFR gene is present in $10 \%$ of the cases, but gene amplification and overexpression is present in almost $30 \%$ and $90 \%$ of cases respectively, allowing for the triggering of multiple intracellular signaling cascades that activate cell proliferation and survival, invasion, metastasis, and resistance to radiotherapy, all mechanisms promoting tumor aggressiveness [16].

Overexpression and mutation of transcription factors as STAT3 involved in EMT, proliferation, apoptosis, and inflammation, assures self maintenance and renewal of cancer stem cells which helps in progression of cancer cells $[17,18]$.

Epigenetic changes most commonly associated to HNSCC are DNA methylation, histone modification, microRNA, and small interfering RNA.

\section{Stromal components of TME that impact on aggressiveness}

They are mainly cancer associated fibroblasts, cells deputed to immune response, metabolism, angiogenesis, inflammation, hypoxia and epithelial mesenchymal transition (EMT).

\section{Cancer associated fibroblasts (CAF)}

Fibroblasts, normally residing in the stroma underlying the epithelium of the upper aerodigestive tract, are responsible for the production of the basal membrane layer, collagen IV, laminin, and numerous cytokine and paracrine signals [19].They are known to coevolve with the tumor, developing a characteristic phenotype called myofibroblast (or active phenotype), due to the cytoplasmic ultrastructural accumulation of contractile proteins such as $\alpha$ - smooth muscle actin (SMA).

Besides the accumulation of specific contractile proteins, CAF produce MMP that directly contribute to remould the extracellular matrix [20].

In HNSCC, CAF show the myofibroblast phenotype associated to expression of integrin $\alpha 6$, critical in the cell-to-cell adhesion maintenance and surface signaling.

It has been demonstrated that upregulation of both $\alpha$-SMA and integrin $\alpha 6$ is correlated with a worsened prognosis in oral can- cer, as complexes $\alpha$-SMA and integrin $\alpha 6$ bind laminins and interact with CDKN1A, altering cell cycle progression [21]. Moreover, CAF secrete and express a variety of factors which promote cell motility by upregulating a number of cytokines, for instance such paracrine motility factor, HGF, CXCL2, and TGF- $\beta$ [22] HGF in turn promotes invasion and angiogenesis [23]. CXCL2 secreted by CAF binds CXXR4, and this interaction plays a role in the upregulation of MMP9, EMT, HIF- $1 \alpha[24]$.

Immune response and immune surveillance

Progression and metastatic dissemination in HNSCC can be objectively linked to the falling and malfunction of host antineoplastic immunity: T lymphocytes and APM cells (antigen presenting machinery cells) play the main role in this process [25].

APM is made up by dendritic cells, Langerhans cells, myeloid dendritic cells and plasmacytoid dendritic cells. All these elements interact with the neoplastic cells in a complex process which can be affected by down-regulation or loss of the HLA class I molecules expression: low survival rates and presence of regional lymph nodes metastasis in primary laryngeal carcinomas and oropharyngeal squamous cell carcinomas are associated to this pathway [26]. Moreover, HNSCC can influence and modulate the circulating myeloid and plasmacytoid dendritic cells, so that the number of these cells is significantly lower in the HNSCC patients; this phenomenon seems to be reversible, for instance the number of circulating cells increases after removal of the tumor.

Langerhans cells (LC) are a particular type of dendritic cells representing 2 - $8 \%$ of the intraepidermal cells in the upper respiratory and digestive mucosa: a strong infiltration of LC cells, distributed both inside and outside the neoplastic sheet, is associated with a low incidence in cervical lymph node metastasis and consequently with a better prognosis [27].

Plasmacytoid dendritic cells (PDC) are antigen presenting cells (APM) able to produce large amounts of interferons IFN- $\alpha$ in response to virus, having a reduced antigen capture potential compared to other APM cells. It is demonstrated that HNSCC cells can negatively influence the PDC function with a reduction in the secretion of IFN, through a tumor-induced down regulation of TLR (toll-like receptor) as studied and demonstrated by Hartmann., et al [28]. 
PGE2 and TGF- $\beta$ are two immunosuppressive factors found in tumor tissue. A recent study showed that TGF- $\beta$ synergized with PGE2 inhibited IFN- $\alpha$ and tumor necrosis factor (TNF) production of TLR7- and TLR9-stimulated PDCs [29].

Finally, tumor associated macrophages (TAM) can contribute to the aggressiveness of HNSCC through their direct participation in angiogenesis and tumor progression.

There is a significant association between the number of TAM and microinvasion, micro vessel density and positivity for angiogenic factors such as VEGF [30]; a direct correlation has been also observed between macrophage infiltration and proliferation index evaluated with the analysis of cell cycle (cyclin E a p53) and proliferation (Ki67) markers, suggesting that TAM are linked to tumor progression [31]. In laryngeal and hypopharyngeal carcinomas the presence and concentration of macrophages was higher in areas of fibrin deposition: this may indicate that they participate to stabilize and to remodel the extracellular matrix, facilitating tumor matrix generation and angiogenesis [32]. A significant direct correlation between TAM and lymph node involvement was demonstrated, the higher concentration of TAM being linked not only to lymph node metastases but also to extracapsular spreading [32].

T cells

The response of the immune system against the neoplastic cells is compromised in the presence of functional defects of $\mathrm{T}$ cells, both circulating and tumor infiltrating lymphocytes (TILS) [34].

A number of defects have been observed in T cells isolated from the tumor, including absence or low expression of CD3 zeta chain, decreased proliferation in response to mitogens, inability to kill tumor cell targets, imbalance in the cytokine profile with absence of IL2, evidence of predominant apoptotic features [34]. Moreover, HNSCC cells can autonomously produce TGF- $\beta 1$ and subsequently generate a reduction in the expression of NK cell receptor NKG2D and CD16, inhibiting the biological function of NK cells.

Mature T lymphocytes (T lymphocytes leaving the thymus and reaching lymph nodes and spleen), are activated when contacted by APC (antigen presenting cells), though the sharing of two specific signals:

1. A link between MHC (major histocompatibility complex) on APC and T-cell receptor.

2. Expression of co-stimulatory molecules (CD25 and FOX-
When activated, $\mathrm{T}$ cells differentiate into effector cells (CD4 helper and CD8 cytotoxic), and into memory cells, which are able to induce a rapid immune response in case of second contact with a previously known antigen.

CD4 T cells (Treg) are important in self-tolerance which means induction of tolerance to self antigens. The main immunophenotypic markers used for the identification of T cells are: CD45RO (naïve and memory T cells), CD69 (activated T cells), CD4 (helper T cells), CD8 (cytotoxic T cells), CD25 and FOXP3 (Tregs).

T lymphocytes in patients with HNSCC have evidenced a number of functional deficits at a loco-regional level (peritumoral infiltration), and on a systemic level, as result of mechanisms which lead HNSCC to evade and to suppress the cytotoxic T lymphocyte anti-tumoral response.

Circulating and tumor associated T lymphocytes can show increased apoptosis through different pathways, such as increased expression of FasL on the cell surface of HNSCC, which promotes apoptosis in peritumoral T lymphocytes through a Fas/FasL interaction [35]. Other pathways are also implicated in the T cell apoptosis, via TRAI and TNF $\alpha$ as demonstrated on Jurkat cell lines [36].

Suppression of cytotoxic T lymphocytes response can develop through the increasing expression of PD-1 (programmed death 1 receptor) on $\mathrm{T}$ lymphocytes and its interaction with the corresponding ligand PD-L1 (expressed by neoplastic cells). This binding shows other adverse effects on antineoplastic immunity, for instance decreased secretion of cytotoxic mediators and increased apoptosis of peritumoral and intratumoral T CD8 lymphocytes [37].

This is the base of the modern therapeutic approaches using molecules able to block the interaction PD-1/PD-L1, not yet validated for clinical purposes in head and neck tumors.

The immunophenotypic characterization of TILS in HNSCC has a long story; in the past it has been mainly intended as tool to establish the conditions for experimental therapies with modifiers of biological response (Valente., et al.) [38]; to date, some groups of T cell populations identified by immunohistochemistry (for instance CD4 - CD69, CD4 - CD25, CD4 - FOXP3) seem to have a prognostic significance; however, the role of FOXP3 positive T lymphocytes has not been conclusively defined: to date, this immunophenotypic class of lymphocytes seem to be associated to a better overall survival and free survival in some studies [39]. P3). 
Interaction between angiogenesis, inflammation and hypoxia in TME

Intratumorally hypoxia, considered as $<2.5 \mathrm{~mm} \mathrm{Hg}$ of $\mathrm{O}_{2}$, is a peculiar characteristic of HNSCC, contributing to its aggressiveness through the resistance to treatment, both chemotherapy and radiotherapy [40]. HIF (Hypoxia Inducible Factor) is a transcription factor that interacts with more than 100 genes, and may be considered the most important and immediate adaptative response to hypoxia, being directly associated with poor prognosis. It interacts with $\mathrm{CA} 9$ and influences $\mathrm{pH}$ of TME with subsequent alterations in the uptake of chemotherapeutic drugs, resulting in resistance to treatment.

The micro vessel density, one of the findings of tumor angiogenesis, is for a long time associated to bad prognosis in a number of head and neck tumors [41], through implication of several factors: for instance, lisyl oxidase catalizes the crosslinking of collagen and elastin, and its overexpression increases the microvessel density. Moreover, a number of other factors derived from TME (VEGF, NF$\mathrm{KB}, \mathrm{HIF}-1 \alpha$ ) participate in the process. VEGF binds to its receptor on tumor cells inducing expression of bcl 2 with an anti-apoptotic effect, and of CXCL1 and CXCL8 in a paracrine fashion: by consequence they promote survival and proliferation of endothelial cells [42].

Chronic inflammation contributes to maintenance and progression of disease, through ROS and COX intervention: COX enzymes catalize the production of prostaglandins, in particular PGE2, which promotes angiogenesis and invasion and inhibits apoptosis in cancer cells.

\section{miRNA expression profiles}

A further attempt to identify prognostic groups of patients has been recently reported in a study which includes miRNA expression profiles obtained from metastatic lymph nodes of not HPVcorrelated HNSCC [43]. Huang., et al. have identified three different subtypes of metastatic HNSCC lymph nodes expression through miRNA expression profiles.

Three main groups were defined, as follows:

Immune subtype (Group 1): predominant signalling pathways were related to $\mathrm{T}$ cell receptor, chemokine receptor and pathways participating in the allograft rejection. Consistent with its enrichment of immunological pathways, a higher estimated proportion of CD4 + B cells and CD8 + T cells.
Invasive subtype (Group 2): invasive subtype was associated with a significant worse prognosis, and its gene expression signature was of prognostic value across multiple cancer types.

Main biological pathways in this group being associated to epithelial-mesenchymal transition, apical junction, TGF-beta signalling, angiogenesis, hypoxia, extracellular matrix receptor interaction, regulation of the actin cytoskeleton and focal adhesion, most gene sets involved in the interaction between cell membrane and extracellular matrix.

Metabolic/ proliferative subtype (Group 3) Gene set enrichment analysis (GSEA) was used to understand the distinct biological pathways characterizing each subtype, defined as follows.

Group 1, immune subtype: predominant active pathways were related to $\mathrm{T}$ cell receptor and chemokine receptor signalling pathway. Consistent with this enrichment of immunological pathways, a higher estimated proportion of CD4 + B cells and CD8+ T cells was present.

Group 2, invasive subtype: associated with a significant worse prognosis, and its gene expression signature was of prognostic value across multiple cancer types.

Main biological pathways in this group being associated to the interaction between epithelial cells and mesenchymal cells through epithelial-mesenchymal transition (EMT), apical junction, TGF-beta signalling, angiogenesis, hypoxia, extracellular matrix receptor interaction, regulation of the actin cytoskeleton and focal adhesion.

Group 3, metabolic subtype: predominant signalling pathways being related to MYC targets, basal transcription factors, and alterations in the mismatch repair genes complex.

\section{Conclusion}

This prognostic classification coming from the translational research could be applied to many other metastatic solid cancer, main problem remaining the many faces of tumoral heterogeneity.

\section{Bibliography}

1. Slaughter DP., et al. "Field cancerization in oral stratified squamous epithelium: clinical implications of multicentric origin". Cancer (1953): 963-968.

2. Curry JM., et al. "Tumor microenvironment in head and neck squamous cell carcinoma”. Seminar in Oncology (2014): 41-42. 
3. Bankfalvi A., et al. "Deranged expression of the E-cadherin/ beta-catenin complex and the epidermal growth factor receptor in the clinical evolution and progression of oral squamous cell carcinomas". Journal of Oral Pathology and Medicine 31 (2002): 450-457.

4. Andrews NA., et al. "Expression of the E-cadherin-catenin cell adhesion complex in primary squamous cell carcinomas of the head and neck and their nodal metastases". British Journal of Cancer 75 (1997): 1474-1480.

5. Ramos DM., et al. "Expression of integrin beta 6 enhances invasive behavior in oral squamous cell carcinoma”. Matrix Biology 21 (2002): 297-307.

6. Kunishi M., et al. "Down-regulated expression of CD44 variant 6 in oral squamous cell carcinomas and its relationship to regional lymph node metastasis". International Journal of Oral and Maxillofacial Surgery 26 (1997): 280-283.

7. Oliveira DT., et al. "Expression of CD44 variant exons by primary and metastatic oral squamous carcinomas". Journal of Oral Pathology and Medicine 27 (1998): 303-307.

8. 0-Charoenrat P., et al. "Differential modulation of proliferation, matrix metalloproteinase expression and invasion of human head and neck squamous carcinoma cells by c-erbB ligands". Clinical and Experimental Metastasis 17(1999): 631-639.

9. O-Charoenrat P., et al. "Overexpression of epidermal growth factor receptor in human head and neck squamous carcinoma cell lines correlates with matrix metalloproteinase- 9 expression and in vitro invasion". International Journal of Cancer 86.3 (2000): 307-317.

10. Vigneswaran N., et al. "Variable expression of cathepsin B and D correlates with highly invasive and metastatic phenotype of oral cancer". Human Pathology 31 (2000): 931-937.

11. Gandour-Edwards R., et al. "Predictive value of cathepsin-D for cervical lymph node metastasis in head and neck squamous cell carcinoma". Head Neck 21 (1999): 718-722.

12. Di Renzo MF., et al. "Somatic mutations of the MET oncogene are selected during metastatic spread of human HNSC carcinomas". Oncogene 19 (2000): 1547-1555.

13. O-charoenrat P., et al. "The role of c-erbB receptors and ligands in head and neck squamous cell carcinoma". Review Oral Oncol 38 (2002): 627-640.
14. Lin SY., et al. "TP53 regulates the migration of mesenchymal stromal cells in response the tumor microenvironment through both CXCL12 -dependent and independent mechanism". International Journal of Oncology 43 (2013): 1817-1823.

15. YoshidA $R$ and Nagatama $M$ "The pathological significance of NOTCH 1 in oral squamous cell carcinoma". Lab Invest 93.10 (2013): 1068-1081.

16. Sheu JJ., et al. "Functional genomic analysis identified epidermial growth factor receptor activation as the most common genetic event in oral squamous cell carcinoma". Cancer Research 69 (2009): 2568-2576.

17. Geiger J., et al. "The STAT3 pathway as a therapeutic target in head and neck cancer: Barriers and innovations". Oral Oncology 56 (2016): 84-92.

18. Squarize C., et al. "Molecular Cross-talk between NFkB and STAT3 pathways in head and neck squamous cell carcinoma". Neoplasia 8 (2006): 733-746.

19. Wheeler SE., et al. "Enhancement of head and neck squamous cell carcinoma proliferation, invasion and metastasis by tumor-associated fibroblasts in preclinical models". Head and Neck (2013).

20. Franco OE., et al. "Cancer associated fibroblasts in cancer pathogenesis". Seminars in Cell and Developmental Biology 21 (2010): 33-39.

21. Lim KP., et al. "Fibroblasts gene expression profile reflects the stage of tumor progression in oral squamous cell carcinoma". The Journal of Pathology 423 (2011): 269-269.

22. Leef T. "Molecular communication between tumor-associated fibroblasts and head and neck squamous cell carcinoma". Oral Oncology 49 (2013): 381-386.

23. Knowles., et al. "HGF and c-MET participate in paracrine tumorigenic pathways in head and neck squamous cell cancer". Clinical Cancer Research 15 (2009): 3740-3750.

24. IshikawaT., et al. "Hypoxia enhances CXCR4 expression by activating HIF1 in oral squamlous cell carcinoma". Oncology Reports 21 (2009): 707-712.

25. Duray A., et al. "Immune suppression in head and neck cancers: a review". Clinical and Developmental Immunology (2010): 701657. 
26. Ogino T., et al. "HLA class I antigen down-regulation in primary laryngeal squamous cell carcinoma lesions as a poor prognostic marker". Cancer Research 66.18 (2006): 9281-9289.

27. Yilmaz T., et al. "Prognostic significance of Langerhans cell infiltration in cancer of the larynx". Journal of Otolaryngology Head and Neck Surgery 139 (2005): 309-316.

28. Hartmann E., et al. "Identification and functional analysis of tumor-infiltrating plasmacytoid dendritic cells in head and neck cancer". Cancer Research 63.19 (2003): 6478-6487.

29. Bekeredjian-Ding I., et al. "Tumour-derived prostaglandin E and transforming growthfactor- $\beta$ synergize to inhibit plasmacytoid dendritic cellderived interferon- $\alpha$ ". Immunology 128.3 (2009): 439-450.

30. Li C., et al. "Infiltration of tumor-associated macrophages in human oral squamous cell carcinoma". Oncology Reports 9.6 (2002): 1219-1223.

31. Ritta M., et al. "Cell cycle and viral and immunologic profiles of head and neck squamous cell carcinoma as predictable variables of tumor progression". Head and Neck 31.3 (2009): 318-327.

32. Bardos H., et al. "Fibrin deposition in squamous cell carcinomas of the larynx and hypopharynx". Thrombosis and Haemostasis 80.5 (1998): 767-772.

33. Marcus B., et al. "Prognostic factors in oral cavity and oropharyngeal squamous cell carcinoma: the impact of tumor-associated macrophages". Cancer 101.12 (2004): 2779-2787.

34. Duray A., et al. "Immune suppression in head and neck cancers: a review". Clinical and Developmental Immunology (2010): 701657.

35. Gastman BR., et al. "Fas ligand is expressed on human squamous cell carcinomas of the head and neck, and it promotes apoptosis of T lymphocytes". Cancer Research 59.20 (1999): 5356-5364.

36. Kassouf $\mathrm{N}$ and Thornhill $\mathrm{MH}$. "Oral cancer cell lines can use multiple ligands, including Fas-L, TRAIL and TNF- $\alpha$, to induce apoptosis in Jurkat T cells: possible mechanisms for immune escape by head and neck cancers". Oral Oncology 44.7 (2008): 672-682.

37. Cho Yoon Lee Hong Hong "Relationship between the expression of PDL-1 and tumor infiltrating lymphocytes in oral squamous cell carcinoma". Oral Oncology 47 (2011): 1148 -1153.
38. Valente G., et al. "Infiltrating leukocyte populations and T-lymphocyte subsets in head and neck squamous cell carcinomas from patients receiving perilymphatic injections of recombinant Interleukin 2. A pathologic and immunophenotypic study". Modern Pathology 3 (1990): 702-708.

39. De Ruiter EJ., et al. "The prognostic role of tumor infiltrating T-lymphocytes in squamous cell carcinoma of the head and neck: A systematic review and meta-analysis". Oncoimmunology 6.11 (2007): e13561.

40. Li JZ., et al. "Hypoxia in head and neck squamous cell carcinoma”. ISRN Otolaryngol (2012).

41. Liang X Yang D Ho J., et al. "Hypoxia inducible factor alfa expression correlates with vascular endothelial growth factor$\mathrm{C}$ expression and lympahngiogenesis/angiogenesis in oral squamous cell carcinoma". Anticancer Research 28 (2008): 1659-1666.

42. Karl E., et al. "Unidirectional crosstalk between Bcl2-xL and Bcl2 enhances the angiogenic phenotype of endothelial cells". Cell Death and Differentiation 14 (2007): 1657-1666.

43. Huang L., et al. Molecular Classification of Lymph Node Metastases Subtypes Predict for Survival in Head and Neck Cancer (1884).

\section{Volume 3 Issue 11 November 2019}

(C) All rights are reserved by Gabriela Alejandra Iasi and Guido Valente. 